\title{
Mechanism of friction reduction of unsaturated fatty acids as additives in diesel fuels
}

\author{
Jean Michel MARTIN ${ }^{1, *}$, Christine MATTA ${ }^{1}$, Maria-Isabel De Barros BOUCHET ${ }^{1}$, Cyrielle FOREST $^{1}$, \\ Thierry Le MOGNE ${ }^{1}$, Thomas DUBOIS ${ }^{2}$, Michael MAZARIN ${ }^{2}$ \\ ${ }^{1}$ Laboratory of Tribology and System Dynamics, Ecole Centrale de Lyon, 69134 Ecully, France \\ ${ }^{2}$ TOTAL, CReS, BP22, 69360 Solaize Cedex, France \\ Received: 07 June 2013 / Revised: 02 August 2013 / Accepted: 05 August 2013 \\ C The author(s) 2013. This article is published with open access at Springerlink.com
}

\begin{abstract}
This paper investigates the effect of unsaturated fatty acid additives on the lubricating properties of a biodiesel, composed of a blend of fatty acid methyl esters (FAMEs) and a conventional diesel fuel. The presence of fatty acids as additives for biodiesel improves their lubricating properties at elevated temperature conditions. The formation of a protective lubricating tribochemical film on the steel contact surfaces has been evidenced. Elevated temperature conditions and possibly oxygen are needed to form this film that reduces friction and limits wear. Several analytical tools have revealed the insulating nature of the formed tribofilm and its chemical properties. Friction-induced reticulation is thought to play a major role in the growth of this insulating film.
\end{abstract}

Keywords: biodiesel; low friction; fatty acids; tribochemistry; surface analysis

\section{Introduction}

In the last decades, fuel economy and long-term impact of exhaust greenhouse gas emissions on the environment have become issues of particular concern, especially for the automotive industry. Development of new fuels directly from renewable natural raw materials and the design of new mechanical components for ever more efficient combustion engine (lightweight body structures, direct-injection systems for engines) [1-3] are becoming crucial steps in order to meet the current economical and environmental concerns. As an example, new biodiesel such as BTL need the presence of lubricity additives to enhance their own natural lubricating properties and optimize the fuel economy character $[4,5]$.

Nowadays, the lubricating properties of biodiesel formulated for fuel injections systems [4-6] are mainly conducted with high-frequency reciprocating rig (HFRR) according to the international standards [7-9].

\footnotetext{
* Corresponding author: Jean Michel MARTIN.

E-mail: jean-michel.martin@ec-lyon.fr
}

Meanwhile, it would be interesting to evaluate the friction properties of the biofuels in different stress conditions in order to investigate their impact on other mechanical parts of the engine where they may be present, together with the lubricant oil, and may act on the long term. This is the case of piston ring/cylinder liner contacts where the sliding speed in the relative motion varies in a broad range and the contact pressure is moderate leading to the existence of mixed and boundary lubrication regimes.

This paper investigates the effects of fatty acid additives on the lubricating properties of biodiesel fuels containing fatty acid methyl esters (FAMEs). FAMEs are produced by a trans-esterification process where glycerol in triglycerides is replaced by methanol [10] and constitute the major components of so-called biodiesel. They can be used directly as a substitute for diesel fuels, or blended in conventional diesel fuel with $\mathrm{BX}$ denomination ( $X$ gives the volume percent of FAME in the final blend) [10]. FAMEs are molecules composed of a polar head and a long fatty acid chain and therefore provide good mixed and boundary 
lubrication effects even when blended at very low concentrations [10]. In this paper, the use of fatty acids as additives in biodiesel to further improve their lubricating properties was investigated in different temperature conditions. A special attention was paid to the formation of any protective lubricating tribofilm on the steel contact surfaces, reducing friction and limiting wear and to its mechanism of action.

\section{Experimental}

\subsection{Friction test experiments}

For tribological tests, a reciprocating cylinder-on-flat tribometer was used in order to simulate the piston ring/cylinder on liner contact geometry and dynamics in thermal engines. Friction pairs were made of AISI 52100 steel and both sliding surface had a mirror surface finish (Ra equal to $2 \mathrm{~nm}$ ). The wear track was $5 \mathrm{~mm}$ long and the sinusoidal sliding speed was $70 \mathrm{~mm} / \mathrm{s}$ at its maximum value (corresponding to a frequency of $7 \mathrm{~Hz}$ ). A normal load of $50 \mathrm{~N}$ was applied ensuring an initial maximum contact pressure of $270 \mathrm{MPa}$, which is in good agreement with practical values. Two temperatures were investigated: $40{ }^{\circ} \mathrm{C}$ and $100{ }^{\circ} \mathrm{C}$. Using diesel fuels as lubricants in the tribometer, it is not possible to use higher temperatures because of strong evaporation. The test duration was 2,000 cycles and all tests were reproduced three times in order to have a good repeatability. The diesel fuel solution was deposited in a small reservoir surrounding the contact area and the two surfaces (cylinder and flat) were completely immersed in the solution prior to the test. The temperature of the whole system was controlled by a thermocouple. At $100{ }^{\circ} \mathrm{C}$, evaporation of diesel fuel is quite small during the friction test and there was no need to replenish the reservoir during the test.

A petroleum diesel (B0) and a biodiesel (B7) composed of a conventional diesel fuel blended with $7 v / v \%$ of FAMEs were used. Single and mixture of fatty acid additives were added to diesel at a concentration of $800 \mathrm{mg} / \mathrm{kg}$. First, we studied a commercial additive package used as a friction modifier (FM1) containing a mixture of saturated and unsaturated fatty acids.
Afterwards, we studied four pure fatty acids with a chain length composed of 18 carbon atoms: stearic acid (C18:0), oleic acid (C18:1), linoleic acid (C18:2) and linolenic acid (C18:3), respectively. All chemicals are from Sigma-Aldrich. For a better understanding, we used methyl mono-oleate (Sigma-Aldrich) to model the FM1 additive. Considering the very low viscosity of the diesel fuel compared with engine oils, lubrication was operated in the boundary regime.

\subsection{Surface Analyses}

After friction tests, the flat samples were washed with B0 in an ultrasonic bath to eliminate excess fatty acids from the surface. Then, surface analyses were carried out within the wear track to characterize the tribofilm and outside the track to have a reference. Techniques used are X-ray photoelectron spectroscopy (XPS), IR spectroscopy and atomic force microscopy (AFM).

The XPS technique allows to characterize the surface chemistry and to connect it with the observed tribological behavior. The monochromatic X-ray used was an $\mathrm{Al} \mathrm{K} \alpha \mathrm{X}$-ray radiation, the electron spectrometer detector was a VG 220I (Thermoelectron, France) and the diameter of the X-ray beam was $1.3 \mathrm{~mm}$. The photopeaks were fitted using a Shirley background, and the quantification was made with the Scofield table of sensibility factors.

The IR technique used in this study was the polarization modulation-infrared reflection- adsorption spectroscopy (PM-IRRAS). It is used for the characterization of thin films or monolayers on metal substrate, because it has the advantage of high surface sensitivity, and of the surface selection rule. In addition, the advantage over the conventional IRRAS mode is that modulated reflectivity is independent on the isotropic adsorption from gas or bulk water. Consequently the interfering effect of water vapor and carbon oxide can fairly be eliminated. The PM-IRRAS spectra were detected from 4 different regions of the tribofilm to check the reproducibility of the measurements.

The AFM observations and measurements were conducted to estimate the film aspect and thickness. A VEECO ${ }^{\circledR}$ CP-II atomic force microscope was used in tapping mode with a sharp silicon probe. 


\section{Results and discussion}

\subsection{Friction results}

In Fig. 1, a friction test carried out in presence of additivated biodiesel B7 (B0 containing $7 v / v \%$ of FAMEs and $800 \mathrm{mg} / \mathrm{kg}$ of the commercial friction modifier FM1) is presented. The results at $40{ }^{\circ} \mathrm{C}$ are compared with those obtained at $100{ }^{\circ} \mathrm{C}$. Figure 1 shows that increasing temperature leads in these conditions to a drastic decrease of friction by a factor 2 after 100 cycles and the friction coefficient stabilizes at about 0.054 , a very low value under boundary lubrication regime. Similar results were obtained with several other diesel fuels provided by the same refinery. Figure 1 also shows optical micrographs of the friction track at the end of the tests after rinsing with neat diesel fuel. It is interesting to notice that at $100{ }^{\circ} \mathrm{C}$, there are no visible scratches and colored film in the center of the track where the speed is maximum. Only wear is visible at the two extremities where the sliding speed is small and the cylinder is changing of direction.

This result shows that friction at $100{ }^{\circ} \mathrm{C}$ is associated with the formation of a tribofilm that reduces both friction and wear considerably. If the friction reduction was due to physical adsorption of fatty acids and according to the traditional explanation, the effect of heating would have led to an increase of friction due to desorption of the molecules from the surface. Based on these results, it looks more that a tribochemical reaction is involved.

As FM1 is composed of several fatty acids, some extra experiments were carried out in order to investigate the respective role of each component of FM1 additive (saturated and unsaturated C18 fatty acids). It is to be noted that linolenic acid is not present in the FM1 additive but is also studied in order to better understand the effect of the increasing number of double bonds. First of all, each fatty acid was tested as a friction modifier inside a B0 diesel fuel.

Figure 2 summarizes the results obtained at $100{ }^{\circ} \mathrm{C}$ with the different mixtures. FM1 is the commercial package of fatty acids already tested in Fig. 1 and its performance is compared to those of pure fatty acids introduced at a concentration of $800 \mathrm{mg} / \mathrm{kg}$ inside the B0 diesel. The friction coefficient is recorded at the steady state value at the end of the test. First of all, the results show that regardless of its chemical structure, the addition of a fatty acid to B0 helps to reduce the friction occurring during the test. Linoleic acid gives the best performance in terms of friction reduction whereas oleic acid leads to the worst ones. Based on these results, it is somehow difficult to draw tendencies on the effect of the number of double bonds on the lubricity performances of the different fatty acids.

Figure 3 displays similar results as in Fig. 2 but in the presence of the biodiesel B7 instead of the B0. We

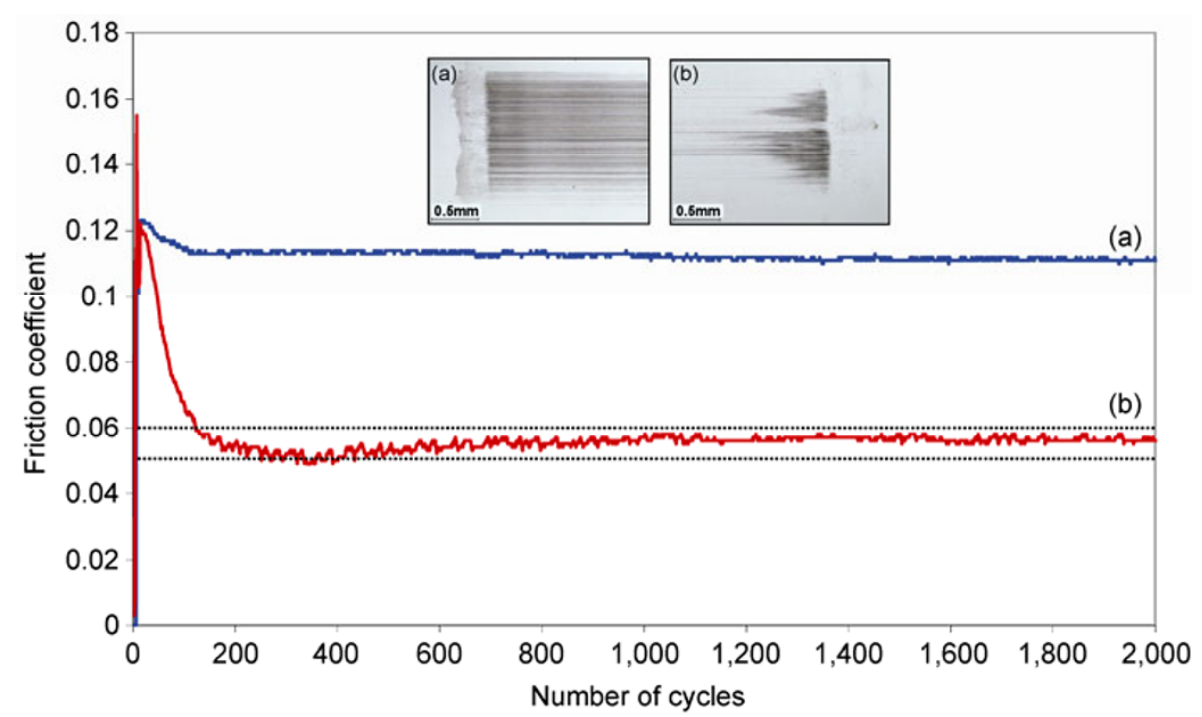

Fig. 1 Friction coefficient as a function of the number of cycles and optical micrographs of flat wear scars. AISI 52100 steel couple lubricated by B7+800 mg/kg FM1 @ (a) $40{ }^{\circ} \mathrm{C}$ and (b) $100{ }^{\circ} \mathrm{C}$. This experiment was repeated three times and gave similar results. 


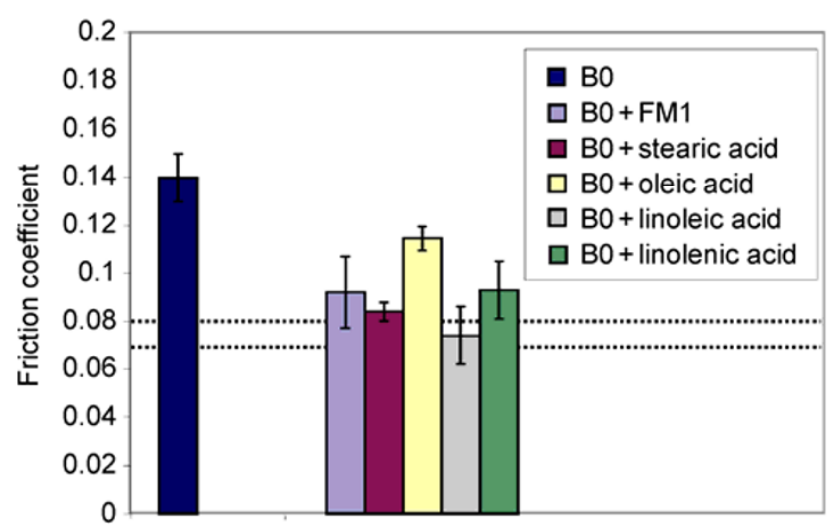

Fig. 2 Steady state friction coefficient as a function of lubricant mixtures in B0 diesel fuel (AISI 52100 steel friction pairs at $100{ }^{\circ} \mathrm{C}$ ).

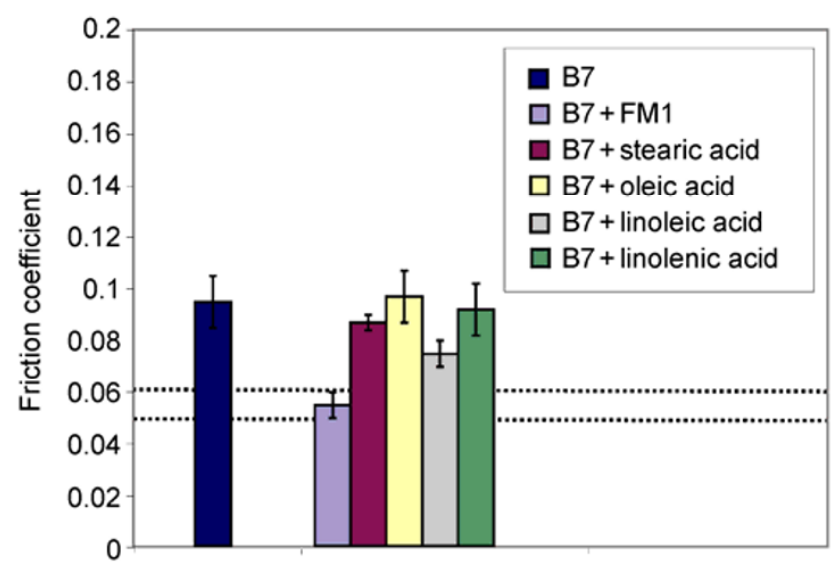

Fig. 3 Steady state friction coefficient vs. lubricant mixtures in B7 biodiesel (52100 steel friction pairs at $\left.100{ }^{\circ} \mathrm{C}\right)$. remind that $\mathrm{B} 7$ is the mixture of $\mathrm{B} 0$ with $7 v / v \%$ of FAMEs. First, it is shown that the presence of FAMEs alone decreases the friction compared to B0. The results also show a strong synergistic effect between FAMEs and FM1 (this was actually the case depicted in Fig. 1) but this effect was not observed with the pure fatty acids taken separately. It is also noted that the respective performances of the pure fatty acids do not seem affected by the presence of the FAMEs since the efficiency classification is the same as that previously shown with B0.

\subsection{Tribofilm analysis}

Figure 4 shows XPS spectra of carbon (C 1s) obtained inside the wear track (no visible wear in optical micrograph in (b)) and outside the wear scar in (a), respectively. The friction test has been performed at $100{ }^{\circ} \mathrm{C}$ with a mixture of B7 and the commercial additive FM1 (mixture of fatty acids, both saturated and unsaturated). Before XPS analysis, the worn surface was cleaned with B0 and dried in air afterwards. First, we observed significant charge effects in the XPS spectrum performed inside the wear scar, indicating that an insulating but transparent to light layer is covering the worn steel surface. Outside the wear scar, there are no charge effects and the signature of adsorbed fatty acids is visible by the presence of the

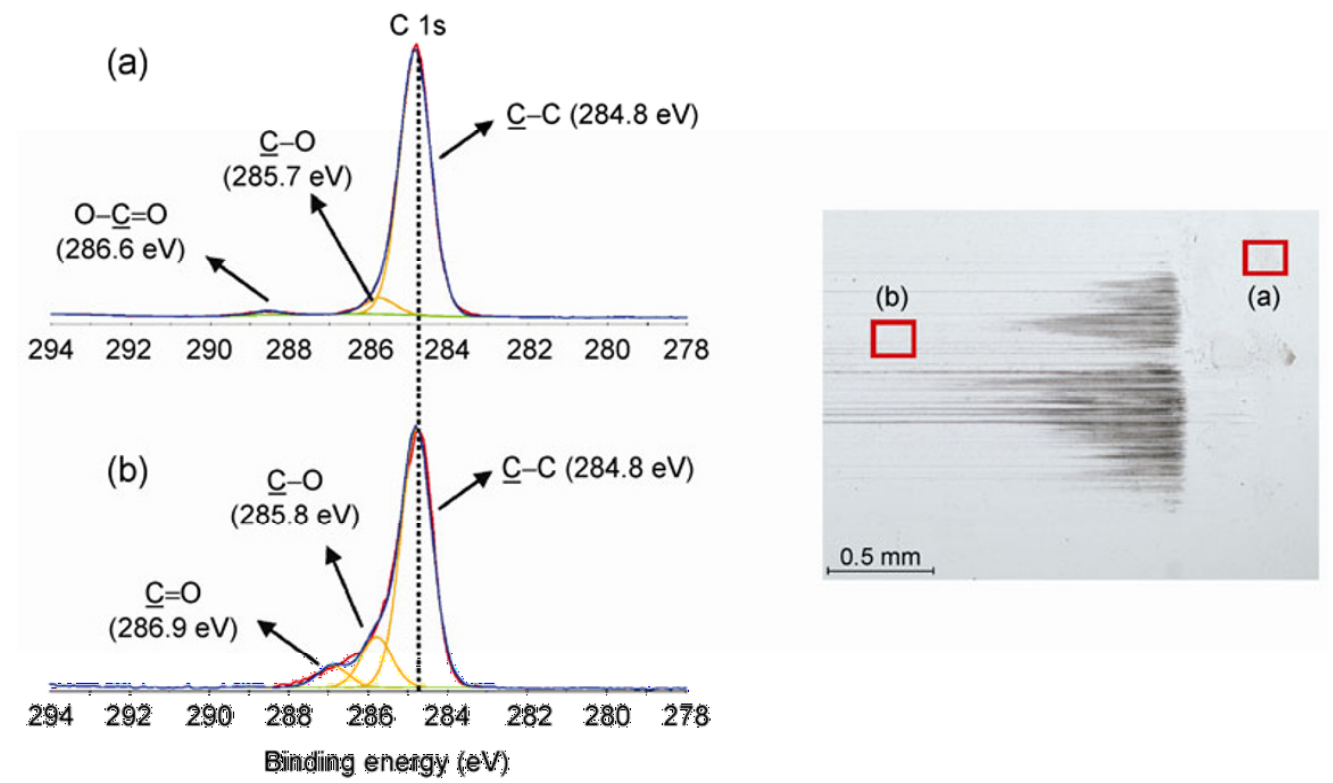

Fig. 4 XPS carbon (C 1s) spectra of (a) outside and (b) inside, the tribofilm formed in presence of FM1 in B7 biodiesel at $100{ }^{\circ} \mathrm{C}$. 
carboxylic peak $(\mathrm{O}-\mathrm{C}=\mathrm{O})$ at $288.6 \mathrm{eV}$ (compared with the $\mathrm{C}-\mathrm{C}$ peak centered at $284.8 \mathrm{eV}$ ). Inside the wear scar, the shape of the carbon peak has changed: The carboxylic contribution has practically disappeared and a new peak at $285.8 \mathrm{eV}$ is detected. This new peak is attributed to $\mathrm{C}-\mathrm{C}-\mathrm{O}$ bonding. Thus, a new compound has been formed inside the wear scar and is responsible for friction reduction of this particular biodiesel formulation. Moreover it can be noted that this tribofilm materiel is not soluble in diesel fuels even after several washes of the surface.

XPS has some limitations to characterize organic species containing only carbon, oxygen and hydrogen elements and the technique is not sensitive to carbon hybridization (presence of double bonds). Consequently we performed complementary analyses by IR spectroscopy. Figure 5(a) shows an ATR-IR spectrum recorded with the pure FM1 additive. The spectrum displays the signature of carbon double bonds $(\mathrm{C}=\mathrm{C})$ at $3,009 \mathrm{~cm}^{-1}$ that is well present in the backbone of unsaturated fatty acids. Methyl and carbonyl groups coming from acid functional groups are also present on the spectrum. The PM-IRRAS spectrum carried out inside the tribofilm is drastically different. First the unsaturation at $3,009 \mathrm{~cm}^{-1}$ has disappeared and a large peak between 1,000 and $1,200 \mathrm{~cm}^{-1}$ has grown. This large peak corresponds to the presence of functional ester groups $(\mathrm{O}=\mathrm{C}-\mathrm{O}-\mathrm{X})$ where $X$ can be iron or an organic chain. This is in good agreement with the XPS results.

Both XPS and IR analyses clearly indicate that a complex tribochemical reaction has occurred to form the tribofilm material on the surface and that the result looks more like a reticulation implying unsaturated fatty acids from the FM1 additive and FAMEs from B7 and possibly oxygen dissolved in the lubricant. Therefore we can understand that the temperature is important for the reaction to proceed. The effect of oxygen from environmental atmosphere may also play a role in the chemical process.

In addition, we used AFM imaging to visualize the tribofilm because it is not visible by optical microscopy. Figure 6 presents AFM images generated inside the wear scar and compared with outside. AFM images clearly show the presence of pads of tribofilm material inside the wear track. These pads are about $10 \mu \mathrm{m}$ wide and their thickness is around $0.1 \mu \mathrm{m}$. Outside the wear track no pads were detected. The thickness profile confirms that no wear has occurred and that the tribofilm is superimposed on the virgin steel surface.

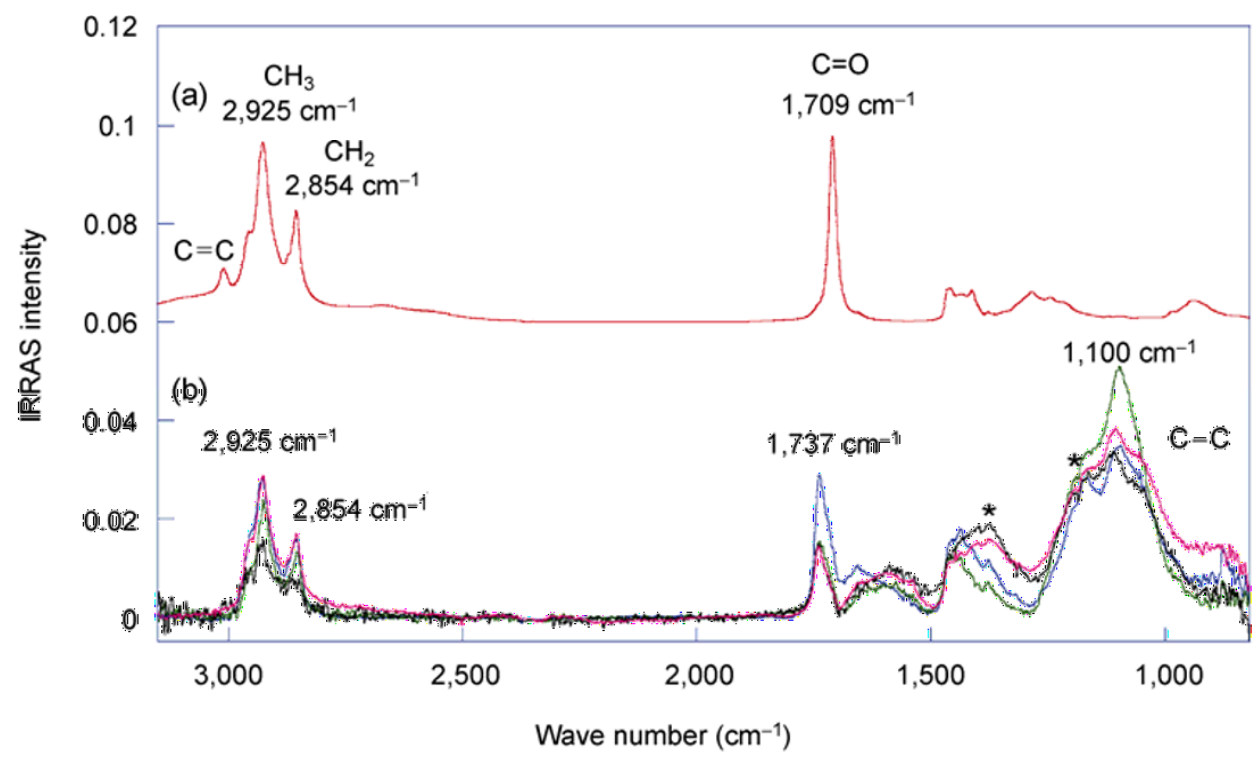

Fig. 5 PM-IRRAS spectra of (a) pure FM1 and (b) the tribofilm formed in presence of FM1 in B7 biodiesel at $100{ }^{\circ} \mathrm{C}$ taken at four different zones. 

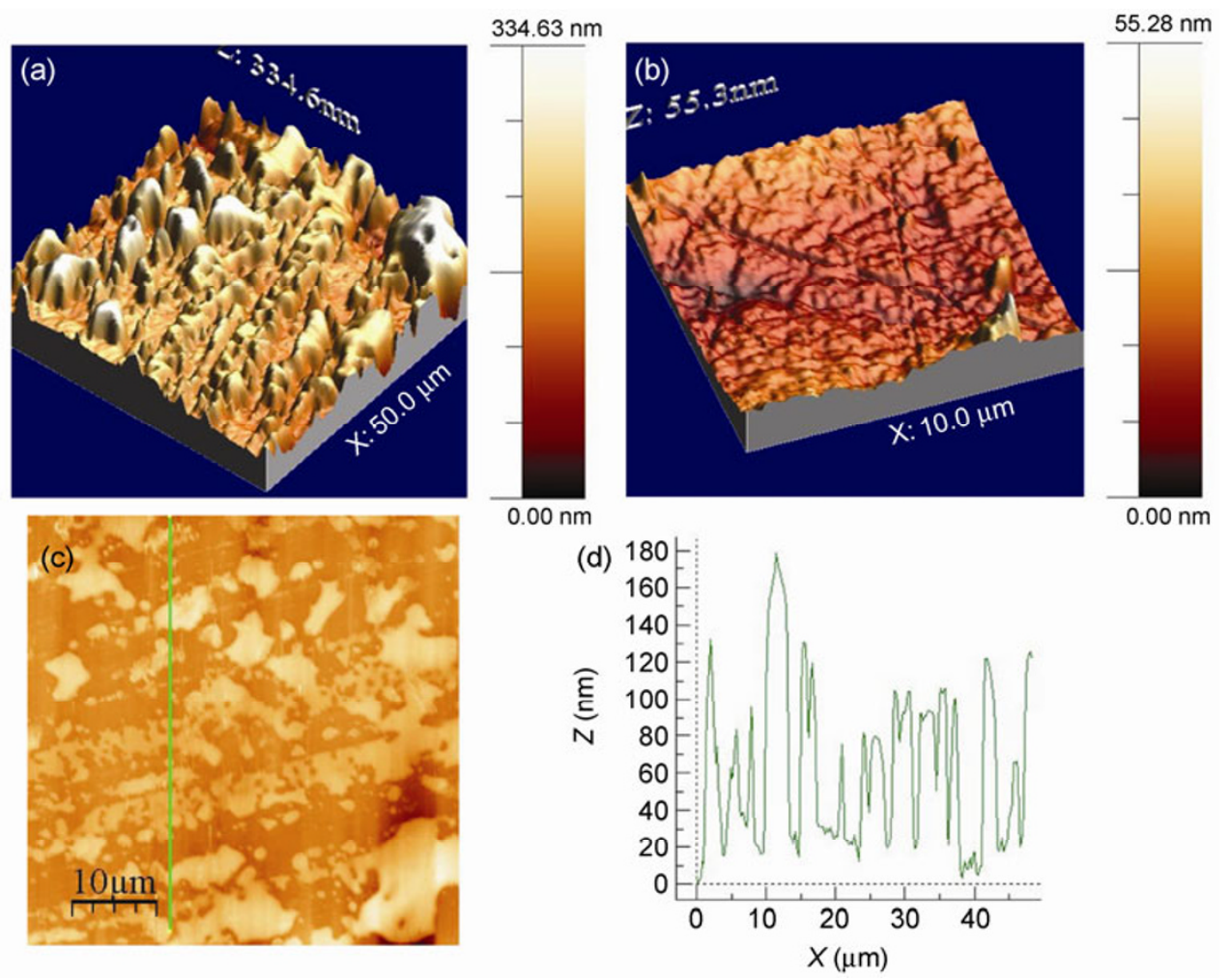

Fig. 6 AFM images of (a) inside, (b) outside the tribofilm formed in presence of FM1 in B7 biodiesel at $100^{\circ} \mathrm{C}$, and (c) AFM line profile inside the wear track.

\section{Conclusions}

In this study, the impact of fatty acids on the lubricity of petroleum diesel and biodiesel fuels was investigated in a steel/steel contact. The tribological conditions were chosen in order to simulate mechanical parameters in the piston ring/cylinder liner system. Two different temperatures were investigated: $40{ }^{\circ} \mathrm{C}$ and $100{ }^{\circ} \mathrm{C}$. The following results were obtained:

1. The B0 diesel fuel alone (with no FAMEs and no fatty acid additives) does not lubricate well the steel contact, whatever the temperature used. Friction is high and wear occurs.

2. The presence of $7 v / v \%$ of FAMEs in B0 (so called B7) lubricates the steel contact to a certain extent. Friction coefficient is reduced by about $30 \%$.

3. The addition of fatty acids in B7 leads to further significant reduction of friction. The FM1 commercial additive (composed of a mixture of different fatty acids) added to B7 offers the best performance (friction is reduced by 2 compared with B0, see Fig. 3). However, the friction reduction only occurs at the highest temperature $100{ }^{\circ} \mathrm{C}$.

4. The effect of pure C18 fatty acids on friction reduction was investigated. This included a comparison of a saturated species (stearic acid) with three unsaturated ones (oleic, linoleic and linolenic acids). All these additives helped to reduce the friction whatever the diesel fuel (B0 or B7). However, a synergistic effect between the biodiesel and some fatty acids has been observed. The mixture of B7 and FM1 produces a friction coefficient of about 0.05 , a very low value under boundary lubrication regime. It is important to notice that diesel fuel is not viscous compared to engine oils and that EHL lubrication is not likely to occur in our case.

5 . The examination of friction tracks by optical microscopy shows that the low friction is associated with the absence of any wear scratches in the center of the wear track. No colored film is visible.

6. The analysis of worn surfaces by XPS first reveals that an insulating film is present in the center 
of the tracks (where friction is low). The XPS spectra of carbon show chemical changes in the tribofilm compared with the fatty acids composition. The carboxylic group is no more present.

7. The analysis of tribofilm by PM-IRRAS gives more insights in its chemical composition. An important feature is the disappearance of carbon double bonds in the tribofilm and the presence of some esters (iron soaps or/and organic).

8. As a conclusion, it is thought that under the combined effects of friction, temperature and possibly oxygen from air, a complex chemical reaction of all additives occurs on the surface generating a new solid-like species. Because double bonds are implied in the process, it is believed that the basic mechanism of tribochemistry is due to a reticulation process. More analytical work is somehow needed to better characterize the tribofilm and to understand the origin of friction reduction.

\section{Acknowledments}

The authors would like to thank T. Buffeteau and G. Le Bourdon from Molecular Spectroscopy Group at Bordeaux University in France for performing PM-IRAS analyses and interpreting the data.

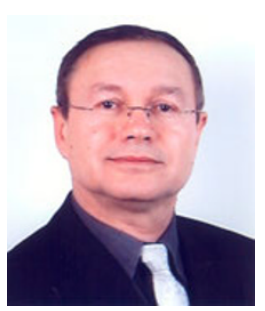

Jean Michel MARTIN. He is a Professor Emeritus at Ecole Centrale de Lyon (University of Lyon, France) since 2011. He obtained his PhD in 1978 from University of Lyon. He is an honorary member of the University Institute of France (IUF). He has more than 35 years of extensive experience in fundamental and applied research in tribology of thin films, diamondlike coatings, boundary lubrication,
Open Access: This article is distributed under the terms of the Creative Commons Attribution License which permits any use, distribution, and reproduction in any medium, provided the original author(s) and source are credited.

\section{References}

[1] Hoshi M. Reducing friction losses in automobile engines. Tribol Int 17(4): 185-189 (1984)

[2] Rosenberg R C. General friction considerations for engine design. SAE Paper 821576 (1982)

[3] Katoh A, Yasuda Y. An analysis of friction techniques for the direct-acting valve train system of a new-generation lightweight 3-liter V6 Nissan engine. SAE Paper 940992 (1994)

[4] Chevron corporation: Diesel fuels technical review, 2007.

[5] Le Pera M. The lubricity of fuels. Tribology \& Lubrication Technology, 2007.

[6] Canter N. Special report: Boundary lubricity additives. Tribology \& Lubrication Technology, 2009.

[7] Diesel fuel-Assessment of lubricity using the HFRR-Part 1: Test method. ISO 12156-1:2006, 2006.

[8] Standard test method for evaluating lubricity of diesel fuels by HFRR. ASTM D6079-11, 2011.

[9] Standard test method for evaluating lubricity of diesel fuels by HFRR by visual observation. ASTM D7688-11, 2011.

[10] Biodiesel handling and use guide-Fourth edition. NREL/ TP-540-43672, 2009.

antiwear and extreme-pressure additives, and surface analysis. He discovered superlubricity of pure $\mathrm{MoS}_{2}$ in ultrahigh vacuum and superlubricity of DLC coatings with $\mathrm{OH}$-containing lubricants, implemented new additive formulations for metalforming and engine applications, and developed new analytical techniques for tribofilm analyses (XPS/AES/ToF-SIMS, XANES, etc.). He has published more than 160 papers in tribology, surface analysis and lubricant engineering and possessed 14 patents. 